\title{
Applicability of the Kinect Sensor in the Rehabilitation of Balance Control in the Elderly: A Pilot Study
}

\author{
Hebert Olímpio Junior ${ }^{1}$, Danielle Romanazzi Lopes ${ }^{1}$, Juan Fernandes de Castro ${ }^{1}$, Plínio dos Santos \\ Ramos ${ }^{1}$, André Gustavo Fernandes de Oliveira ${ }^{2}$ and Agnaldo José Lopes ${ }^{3,}{ }^{*}$ \\ ${ }^{1}$ Faculty of Medical and Health Sciences of Juiz de Fora (SUPREMA), Juiz de Fora, Brazil \\ ${ }^{2}$ Federal University of Juiz de Fora (UFJF), Juiz de Fora, Brazil \\ ${ }^{3}$ Rehabilitation Sciences Post-Graduate Program, Augusto Motta University Center (UNISUAM), Rio de Janeiro, Brazil \\ Corresponding author: Rehabilitation Sciences Post-Graduate Program, Augusto Motta University Center (UNISUAM), Avenida Paris, 72, Bonsucesso, 21041-020, Rio de Janeiro \\ Brazil. Email: agnaldolopes.uerj@gmail.com
}

Received 2018 July 12; Revised 2018 October 19; Accepted 2018 November 02.

\begin{abstract}
Background: Taking into consideration the inversion of the age pyramid in the coming years and the limitations and diseases that predispose the elderly to episodes of falling, it is necessary to develop resources that can address postural balance in this population. The use of virtual rehabilitation, i.e., rehabilitation using electronic platforms, has been increasing due to the increased treatment adherence observed with these methods.

Objectives: This study was conducted to evaluate the effectiveness of a rehabilitation program using the Kinect sensor (KS) on the postural balance of the elderly.

Methods: This was a non-randomized controlled clinical trial in which 10 elderly subjects and 10 younger adults underwent a 10session rehabilitation protocol lasting 20 minutes per session. Each session involved muscle stretching and motor coordination exercises, as well as use of the KS. The period between the evaluations was five weeks, and the sessions were held three times per week. The initial and final evaluations included the Berg Balance Scale (BBS), the Timed Up and Go (TUG) test and the tandem Romberg test. Results: After treatment with the KS, the elderly required a longer time to perform the TUG test and had lower static balance results in the tandem Romberg test compared to younger adults; however, there were no significant differences between the pre- and posttreatment values for these two tests within either group $(\mathrm{P}>0.05)$. The elderly scored lower on the BBS than the younger adults both before and after treatment $(\mathrm{P}=0.0001$ and $\mathrm{P}=0.0006$, respectively). However, the elderly showed a balance gain according to the BBS scores between the pre- and post-treatment evaluations $(\mathrm{P}=0.005)$, which did not occur in the younger adults $(\mathrm{P}=0.31)$. Conclusions: The KS is able to promote improvements in static and dynamic postural balance in the elderly, who reached a condition close to what was observed in young adults. This improvement is evident when evaluating the changes using the BBS.
\end{abstract}

Keywords: Aged, Balance Control, Kinect Sensor, Berg Balance Scale

\section{Background}

Globally, the number of elderly individuals is growing faster than all of the younger age groups. The elderly population -those aged 60 years or older- is expected to more than double by 2050 and more than triple by 2100 , from 962 million worldwide in 2017 to 2.1 billion in 2050 and 3.1 billion in 2100 (1). In this scenario, the growth of the elderly population has become a public health concern because aging brings with it a series of physiological and anatomical changes that predispose people to chronic diseases and functional limitations (1-4). Therefore, possible repercussions, such as falls, can occur frequently in the elderly population. Falls are defined as episodes of imbalance that can cause the elderly to come into accidental contact with the ground or nearby surfaces $(5,6)$. Falls increase rates of morbidity, mortality and hospitalization and can cause injuries that are costly to treat $(7,8)$. Approximately $30 \%$ of falls result in severe injuries in the elderly, and there is a relationship between age and the frequency of episodes of postural imbalance (9). Since one in three elderly individuals suffer falls, it is necessary to develop interventions to promote the maintenance of postural balance in this population (10).

Balancing is an extremely complex process that involves the proper functioning of the vestibular, visual and central and peripheral nervous systems, as well as the responses of the musculoskeletal system to sensory stimuli. These responses are related to the integrity of range of mo-

Copyright (c) 2018, Author(s). This is an open-access article distributed under the terms of the Creative Commons Attribution-NonCommercial 4.0 International License (http://creativecommons.org/licenses/by-nc/4.0/) which permits copy and redistribute the material just in noncommercial usages, provided the original work is properly cited. 
tion, muscle strength and proprioception (11). As a biomechanical strategy for maintaining balance, the individual tries to maintain his or her center of gravity on his or her support base using all possible means of doing so (12-14).

Conventional balance exercises are one of the most preferred exercise options for improving balance in geriatric populations. As an alternative to conventional balance training, virtual reality rehabilitation (VRR) has increasingly been used. VRR is an emerging technology used for the physical restoration of different populations. It is defined as a two- or three -dimensional computerized simulation that gives users the illusion of interactively entering a virtual world in real time (15). During real-time use, it is believed that the central nervous system incorporates sensory augmentation as an additive input, supplementing other sensory information (16).

VRR can promote adaptation of the individual in the proposed training, which is followed by a progression of exercises through varied levels of difficulty offered by the platforms. This model is consistent with the recommendations made regarding exercises for the geriatric population (i.e., starting an activity according to the individuals' capacities and gradually looking for an evolution of functionality) (17). Both randomized and nonrandomized controlled trials have reported positive effects of VRR activities on gait speed, functional balance, and cognition $(18,19)$. In addition, VRR-based balance exercises seem to improve strength, mobility, and functional abilities, while decreasing fears of falling, in community-dwelling older adults (16-19).

Currently, electronic platforms, such as the Nintendo ${ }^{\mathrm{TM}}$ Wii Fit, are being used as tools for VRR in the field of physical therapy (20). VRR leveraging these platforms has shown satisfactory results in various clinical conditions, mainly due to the excellent adherence of individuals to treatment plans $(21,22)$. In addition, in recent years, sensors have been developed that capture movements of the user's entire body and reproduce them faithfully on a screen. One example of this is the Kinect sensor (KS), which was developed by the Microsoft Corporation (20). These sensors allow the user to have the freedom to move without the need for controllers and offer different types of games, with each emphasizing a certain type of motion.

The use of sensors that capture body motion may be an effective option to address the need for new resources to treat postural imbalance, especially since the proposed activities, in addition to being playful, stimulate movements similar to exercise therapies used in balance rehabilitation. Despite being more accurate and reliable compared to other platforms, the KS is still rarely used in VRR of postural balance in elderly subjects (23).

\section{Objectives}

To evaluate the effect of training using body sensors on body balance in the elderly.

\section{Methods}

\subsection{Participants}

Between October 2017 and May 2018, a non-randomized controlled clinical trial was conducted with 16 individuals aged $\geq 60$ years. Individuals who reported previous heart disease, uncontrolled hypertension, visual and/or auditory disorders, neurological disorders and musculoskeletal problems were excluded $(24,25)$. Additionally, a Berg Balance Scale (BBS) score of less than 45 points was also used as exclusion criterion because these individuals have a high risk of falls during walking $(26,27)$. The project was approved by the Research Ethics Committee of the Faculty of Medical and Health Sciences of Juiz de Fora (SUPREMA), Juiz de Fora, Brazil, under number 0015/12 and is in compliance with the provisions of the Declaration of Helsinki. All of the individuals who participated signed consent forms.

We also evaluated a control group that consisted of 10 young adults with a median age of $23(20.1-27.8)$ years, of whom five were men. The median body weight of this group was $65.6(59.8-70.1) \mathrm{kg}$. This group was subjected to the same exclusion criteria as the group of elderly subjects.

\subsection{Intervention}

The treatment protocol consisted of 10 sessions lasting 20 minutes each. During this time, 14 minutes were allocated to the application of stretching exercises, and the remainder was used for rehabilitation with the KS (Microsoft Corporation, Redmond, WA, USA). The period between the evaluations was five weeks, and the sessions were performed three times per week. Before the first session and one day after the last session, participants underwent static and dynamic balance assessments; a resting state was used for these assessments. In this and the other sessions, the participants performed muscle stretching exercises, which were followed by the activity with the KS.

\subsection{Berg Balance Scale}

The BBS is widely used in clinical practice, especially among the elderly and people with neurological disorders in rehabilitation programs. It consists of 14 common static and dynamic balance tasks based on activities of daily living such as standing up, transferring, standing unsupported, reaching and turning. Each task yields a score between zero and four points. If the participant required supervision or an external aid to perform the task, a lower 
score was assigned for the corresponding task. The evaluation was based on the sum of the tasks' points, with a potential maximum score of $56(26,27)$. After the BBS was finished, the participants completed a sequence of specific tests of static and dynamic balance, which also served as a parameter for comparing gains in postural balance (28).

\subsection{Timed Up and Go test}

The Timed Up and Go (TUG) test is performed by having the individual rise from a chair with a backrest, walk three meters and return to the chair. The speed of the execution of the task is established by the participant, as there is no stimulus through verbal commands. For this study, the time taken by the participant to complete this task was measured at the initial evaluation and after the 10 sessions were completed $(29,30)$.

\subsection{Tandem Romberg Test}

The participants held themselves in a standing position with their eyes closed and the calcaneus of their nondominant foot in front of the toes of their dominant foot until they lost their balance or reached the maximum time of one minute $(31,32)$.

\subsection{Muscle Stretching}

After blood pressure measurements were obtained, participants performed muscle stretching exercises to increase their performance during the KS sessions because the flexibility triggered by these exercises is an important tool for adequate postural balance $(13,33)$. The muscle stretches were performed bilaterally and in an activeassisted manner. The stretches included lateral cervical flexion, forward and backward cervical inclination, standing trunk flexion, lateral trunk inclination and rotations in the transverse plane. For the upper limbs, shoulder and elbow flexion, horizontal adduction of the shoulder joint and flexion and extension of the fingers and wrists were performed.

\subsection{Kinect Sensor}

The Stack 'Em Up mode (difficult level) in the Your Shape ${ }^{\mathrm{TM}}$ Fitness Evolved game, which is available on the Xbox 360 platform, was used. The participants were instructed in how to complete the activity and were aided by verbal commands. They positioned themselves in front of the KS and remained in a static position, with their upper limbs raised, simulating holding a board. In the game, colored blocks are dropped onto this board, which must be dropped into an opening on the floor by inclining the trunk laterally, based on the visual stimulus received, to the same side as the opening on the floor, which is simulated on the bottom half of the screen. The floor opens alternately on the right and left sides. Occasionally, burning blocks are dropped, and to put them out, it is necessary to raise the lower limb on the same side as the block that is descending toward the board through hip and knee flexion of $90^{\circ}$.

\subsection{Statistical Analysis}

The distribution of the data was analyzed using the Shapiro-Wilk test along with graphical analysis of the histograms. Because the variables presented as non-Gaussian distributions in at least one of the study moments (before and after intervention with the KS) and/or groups (elderly and younger adults), non-parametric tests were used. Inferential analysis consisted of the Wilcoxon signed-rank test to compare the assessments before and after intervention with the KS within each group (intragroup analysis) and the Mann-Whitney test to compare the results of the elderly with the results of the young adults (intergroup analysis). The values of relative delta between the two moments of the study (before and after intervention with the KS) were calculated as follows:

$\frac{\text { post } K S \text { value }- \text { pre } K S \text { value }}{\text { pre } K S \text { value }} \times 100$

Variables were described in terms of median and interquartile interval values. Differences were considered statistically significant at $\mathrm{P}<0.05$, with $95 \%$ confidence intervals. Analyses were conducted using SAS 6.11 software (SAS Institute, Inc., Cary, NC, USA).

\section{Results}

Of the 16 elderly individuals who were evaluated for inclusion in the study, six were excluded for the following reasons: Visual and/or auditory disorders $(n=2)$, musculoskeletal problems $(n=2)$ and BBS scores of less than 45 points $(n=2)$. The median age of the elderly subjects was $64.5(60.8-68.7)$ years, and five of them were men. The median body weight of this group was $61.7(56.4$ - 66.5) $\mathrm{kg}$, which was not significantly different from that of the control group $(\mathrm{P}=0.13)$.

The results of the body balance tests for the elderly and young adults are shown in Table 1 . Relative to the young adults, the elderly required a longer median time to perform the TUG test both before and after KS training $(\mathrm{P}=0.003$ and $\mathrm{P}=0.015$, respectively). However, there were no significant differences in the TUG test (relative deltas) within either group between the pre- and postintervention analyses (Figure 1). 


\begin{tabular}{|c|c|c|c|}
\hline Variables & Elderly & Younger Adults & PValue $^{c}$ \\
\hline \multicolumn{4}{|l|}{ TUG test, $s$} \\
\hline Before using KS, s & $12(8.8-14.5)$ & $7(6-8.8)$ & $0.003^{\mathrm{c}, \mathrm{d}}$ \\
\hline After using KS, s & $11.5(9.8-13)$ & $7.5(5-10)$ & $0.015^{\mathrm{c}, \mathrm{d}}$ \\
\hline Pvalue $^{b}$ & 0.96 & 0.81 & \\
\hline \multicolumn{4}{|l|}{ Tandem Romberg, s } \\
\hline Before using KS, s & $9.5(4.8-60)$ & $60(60-60)$ & $0.012^{\mathrm{c}, \mathrm{d}}$ \\
\hline After using KS, s & $56(17.8-60)$ & $60(60-60)$ & $0.045^{c, d}$ \\
\hline Relative delta,\% & $275(0-950)$ & $0(0-0)$ & 0.11 \\
\hline \multicolumn{4}{|l|}{ BBS, points } \\
\hline Before using KS, points & $52(49.8-53.3)$ & $56(56-56)$ & $0.0001^{\mathrm{c}, \mathrm{d}}$ \\
\hline After using KS, points & $54.5(54-55.3)$ & $56(56-56)$ & $0.0006^{\mathrm{c}, \mathrm{d}}$ \\
\hline Relative delta, \% & $4.8(3.3-8.1)$ & $0(0-0)$ & $0.0001^{c, d}$ \\
\hline Pvalue $^{\mathrm{b}}$ & $0.005^{b, c}$ & 0.31 & \\
\hline
\end{tabular}

Abbreviation: BBS, Berg Balance scale; KS, Kinect sensor; TUG, Timed Up and Go test.

${ }^{a}$ Values are expressed as median and interquartile interval values.

${ }^{\mathrm{b}}$ Wilcoxon signed-rank test for comparison between the pre- and post-KS intervention points within each group.

${ }^{\mathrm{C}}$ Mann-Whitney test comparing the two groups of individuals.

${ }^{\mathrm{d}}$ Statistical significance $\mathrm{P}<0.05$.

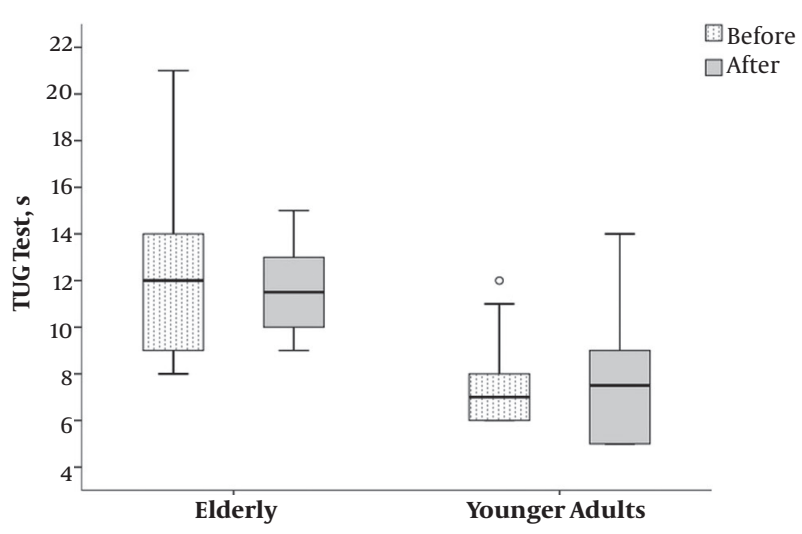

Figure 1. Box plots (median, 1st and 3rd quartiles, minimum and maximum) of the Timed Up and Go (TUG) test. Significant differences were found between elderly and younger adults before $(\mathrm{P}=0.003)$ and $\operatorname{after}(\mathrm{P}=0.015)$ use of the Kinect sensor.

In the tandem Romberg test, the two groups showed different values both before and after the KS intervention ( $\mathrm{P}=0.012$ and $\mathrm{P}=0.045$, respectively). However, there were no significant differences within either group when the differences between the pre-and post-treatment values (relative deltas) were assessed (Figure 2).

When evaluated using the BBS scale, there were significant differences between the elderly and young adults

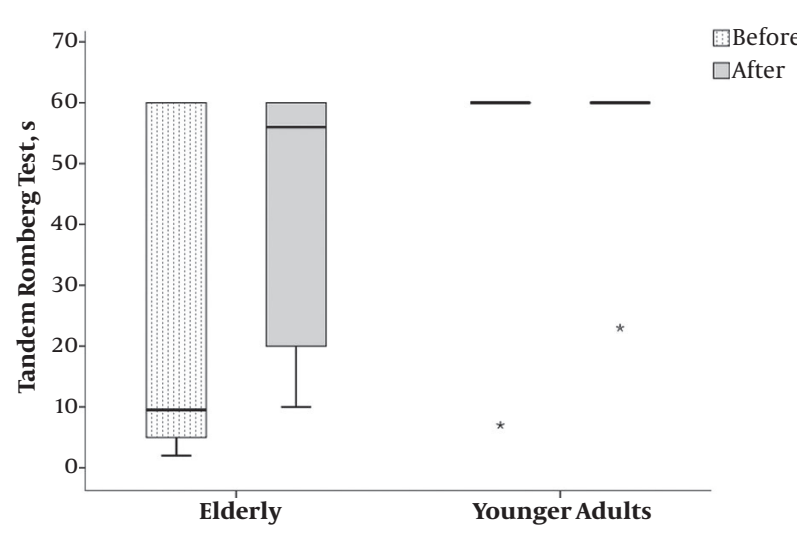

Figure 2. Box plots (median, 1st and 3rd quartiles, minimum and maximum) of the Tandem Romberg test. Significant differences were found between elderly and younger adults before $(\mathrm{P}=0.012)$ and after $(\mathrm{P}=0.045)$ use of the Kinect sensor.

both in the initial evaluation $(\mathrm{P}=0.0001)$ and in the final evaluation $(\mathrm{P}=0.0006)$. When the relative deltas between the pre- and post-treatment BBS scale values were evaluated, a significant difference was only observed in the elderly group $(\mathrm{P}=0.005)$ (Figure 3$)$. 


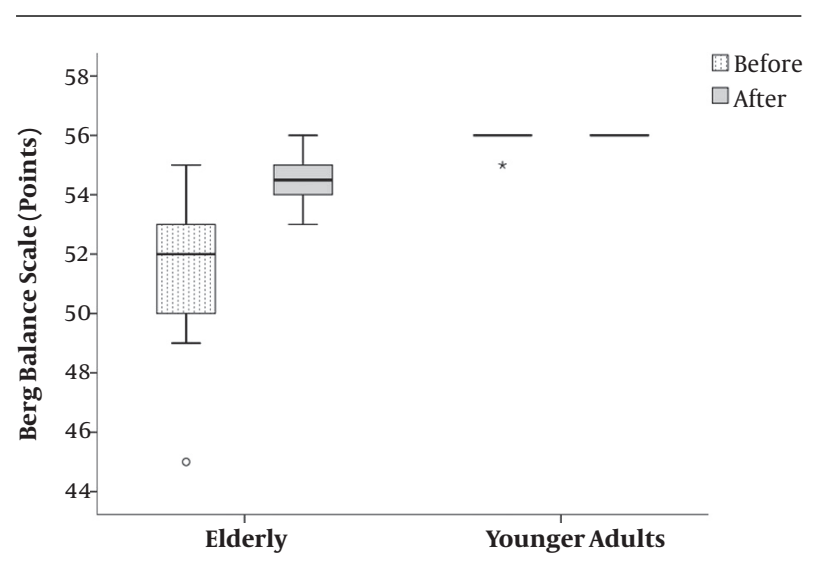

Figure 3. Box plots (median, 1st and 3rd quartiles, minimum and maximum) of the Berg Balance scale. Significant differences were found between elderly and younger adults before $(\mathrm{P}=0.0001)$ and after $(\mathrm{P}=0.0006)$ use of the Kinect sensor.

\section{Discussion}

The main findings of the present study were the significant improvements in static and dynamic balance in the elderly subjects after a rehabilitation program with the KS based on evaluations using the BBS. In addition, the elderly showed an improvement in static balance as measured by the tandem Romberg test, although this improvement only showed a trend toward statistical significance. To our knowledge, this is the first study to show an improvement in postural balance in the elderly after a rehabilitation program using the KS.

In conventional rehabilitation programs, it is very difficult for health professionals to teach patients to optimize their postural balance. In addition, the exercises usually become repetitive and adherence to the interventions gradually decreases, especially in the elderly population. VRR, in this context, can promote the completion of challenging exercises using technological resources that provide auditory, visual and proprioceptive feedback, thereby integrating the systems involved in postural control in a playful way, which consequently results in greater motivation to continue treatment (34). One of the benefits of VRR is the ability of the trainer to vary environmental conditions and give feedback; greater learning is demonstrated with training in virtual rather than physical environments (35). Upon undergoing VRR, the geriatric population exhibits improvements in postural balance even though these vary across types of platforms with regard to intensity, duration, repetitions, and series (36).

In the present study, after VRR using the KS, the elderly subjects did not present with a significant reduction in the time spent performing the tasks in the TUG test. These results are in contrast to those observed by Hara and Shi- mada (37) who used the TUG test to evaluate the dynamic postural balance of elderly subjects after an exercise program performed two to three times per week. The authors observed a reduction in the test execution times after the intervention. Interestingly, Kimura et al. (38) suggested, based on the execution time of the TUG test by the elderly subjects in their study, that age is directly proportional to the time required to complete the test.

Despite disagreements in the literature regarding the determination of a cutoff point in the TUG test to predict the minimum time suggestive of a fall risk, this is one of the tests most commonly used in clinical practice (39). Shumway-Cook et al. (39) proposed that a time greater than 13.5 seconds represents a high risk of falls, while Okumiya et al. (40) established a higher cut-off point of approximately 16 seconds. In the present study, although we did not observe significant differences between the pre- and post-intervention analyses, the reduction of the median from 12 seconds to 11.5 seconds in the test execution time in the elderly may have some clinical importance because there was an increase in the distance between the TUG test results and the cut-off points previously described.

Regarding the static balance assessment, Longridge and Mallinson (41) used the tandem Romberg test and did not find significant differences between young and elderly adults in relation to performance on the test, suggesting that this test is not effective at predicting balance conditions since it is not sufficiently precise in distinguishing individuals from different age groups. Contrary to these findings, we observed a significant difference between the two groups in the tandem Romberg test, with an obvious distinction in the time maintaining balance at the initial evaluation. Importantly, our study showed a trend towards statistical significance in the Romberg tandem test in the elderly after treatment with KS. This outcome is explained by the fact that the Stack 'Em Up mode in the Your Shape ${ }^{\mathrm{TM}}$ Fitness Evolved game intermittently demands lateral trunk flexion and one-leg stance movements, which stimulates straightening and trunk balance reactions. These reactions are also required during the tandem Romberg test, as the body's weight is predominantly supported by ankle inversion and eversion movements when the patient positions one leg anteriorly and in the same line as the other (42). Although it only evaluates static balance and its execution is substantially influenced by the learning effect, the tandem Romberg test is a simple, low-cost test that does not take up too much time during physical examinations and balance control assessments (31).

According to Whitney et al. (43) the BBS is one of the most relevant tests for predicting the risk of falls. This instrument is considered safe and easy to apply in the elderly population because it only requires verbal guidance and 
its equipment costs and supervision requirements during the performance of the tasks are low (26). Park and Lee (44) established through a meta-analysis that cut-off scores between 45 and 49 points had lower heterogeneity for the interpretation of the risk of falls and that the accuracy of the BBS scale was 0.84 based on the groups studied. The VRR intervention using the KS proposed in the present study yielded a gain of 2.5 points in the median score when comparing the pre- and post-intervention analyses (52 vs. 54.5 points). The control of postural balance in the elderly as measured using the BBS in the present study was far from the cut-off points observed in the meta-analysis by Park and Lee (44), including in the studies that considered values of up to 49 points as predictors of a high risk of falls; however, it is worth noting that we used a BBS score of below 45 points as an exclusion criterion in the present study. In agreement with our findings, Yesilyaprak et al. (45) observed that BBS scores improved significantly, with a 3.4point mean change, following VRR in older adults. These authors used VRR exercise sessions through balance training with visual displays and audio feedback.

Using a meta-analysis, Lesinski et al. (46) suggested that the duration of a single training session of 31 - 45 minutes is critical for positive outcomes in the static and dynamic balance of elderly patients. These authors also suggested a frequency of three times per week as being most adequate for achieving therapeutic success. Although our study also used a frequency of weekly sessions similar to that suggested by Lesinski et al. (46), the duration of each session with the KS was five to seven times shorter than that recommended in the rehabilitation of balance using conventional methods (46). This reinforces the importance of playful and motivational training in the geriatric population through VRR.

Hernandez et al. (47) subjected elderly individuals with Alzheimer's dementia to a systematized regimen of physical activity that included dance, stretching and relaxation exercises; each activity session was 60 minutes long, sessions were held three times per week, and the intervention was held over a six-month period. The researchers evaluated balance using the BBS and the TUG test, and they did not observe significant differences between the pre- and post-intervention evaluations in the experimental group. The intervention in our study, on the other hand, showed a significant increase in the BBS score even with a total intervention time 10 times lower than that of Hernandez et al. (47). In addition, there was a significant difference in relation to the lengths of these interventions. It should be noted, however, that Alzheimer's disease presents with neurological deficits that lead to apraxia, hypertonia and postural changes in the medium and long term, and this disease was not reported in the evaluation of any of the volunteers in the present study.

Another aspect worth mentioning is the inversely proportional relationship between age and the BBS score. In a comparative study by Pereira et al. (48), the authors suggest that the BBS is able to discriminate the risk of falls depending on the age of the individuals. This fact was also observed in our study and, additionally, an increase in the sum of the points after the intervention was observed, thus showing a reduction in the risk of falls in the elderly population after the KS intervention.

An additional advantage of VRR in the elderly is improvement in social and family interactions, which may be important in the context of the natural consequences of aging (49). It is worth mentioning that the motor learning achieved through this therapeutic method can be transferred to the activities of daily living, thereby promoting the reintegration of the elderly into the environment (45). These factors extend to institutionalized patients who, although often subjected to conventional methods of the rehabilitation of balance, experience limited stimulation because there is not much variation in the tasks proposed. This condition can be modified with the use of virtual platforms such as the KS (50).

Using the KS, Clark et al. (51) proposed that a threedimensional system for the detection of body positions may be valid as a tool for the rehabilitation of postural balance in the clinical setting. This is confirmed in our study, as we found that the postural control of the elderly subjects improved. Llorenz et al. (52) used the KS as a resource for the rehabilitation of postural control in patients in the spastic phase of stroke recovery; they observed a significant difference between pre- and post-intervention results. One of the relevant aspects of the intervention proposed by the present study is the duration of the sessions, which at only six minutes is relatively short when compared to the previously described clinical trials $(47,51)$.

Other devices that can be used as short-term therapeutic modalities, such as the Wii, also demonstrated an increase in the BBS score. According to Bateni (53), the sessions performed with this device improved postural balance through a four-week protocol with an intervention period of four weeks, with three sessions performed each week. However, the KS allows greater freedom for the lower and upper limbs in relation to other platforms and thus is able to cover a greater number of body functions.

The relevance of our study is the playful approach for the elderly through a treatment protocol with shortduration sessions and the search for an integration of several systems involved in postural balance using a low-cost and widely available device. However, like any study, ours also has its limitations. First, the sample size is relatively small. Second, we did not evaluate the vestibular system 
before the intervention; the vestibular system provides major information on the maintenance of balance, and rehabilitation plans are often designed based on vestibular system disorder. Third, we did not use a more objective method to evaluate balance, such as a force platform. Finally, the participants did not undergo training to perform the sessions with the KS. Despite these limitations, the findings of the present study may serve as a starting point for future trials with subjects with one of the many clinical conditions known to alter postural control.

In conclusion, the treatment protocol involving the KS was able to promote an improvement in postural control in elderly subjects as evaluated using the BBS, and this improvement helped them reach levels close to those observed in younger adults. For the TUG test and tandem Romberg test, there was also improvement in the postural balance of the elderly subjects despite the absence of a significant difference between the pre- and post-intervention values. Thus, our findings suggest that VRR is an interesting resource that promotes positive effects on adherence to treatment and improves clinical outcomes. The use of VRR technology to design games focused on augmenting traditional rehabilitation interventions will likely play an important role in the future treatment of older adults with and without disabilities. Larger clinical studies are needed to establish the efficacy of VRR on the sensorimotor system using various clinical populations.

\section{Footnotes}

Authors' Contribution: Hebert Olímpio Junior contributed to all parts of study and manuscript preparation. Others contributed to data analysis and to the editing and preparation of the manuscript.

Financial Disclosure: We have nothing to declare.

Funding/Support: This research was supported by the Brazilian Council for Scientific and Technological Development (CNPq), Coordination for the Improvement of Higher Education Personnel (CAPES), and the Foundation for Research Support of the State of Rio de Janeiro (FAPERJ).

\section{References}

1. United Nations. World population prospects: The 2017 revision, key findings and advance tables. New York; 2017. Available from: https://esa.un. org/unpd/wpp/.

2. Rodrigues NC, Rauth J. Os desafios do envelhecimento no Brasil. In: Freitas EV PL, editor. Tratado de geriatria e gerontologia. 2nd ed. Rio de Janeiro: Guanabara-Koogan; 2002. p. 106-10.

3. Judge JO, Ounpuu S, Davis R3. Effects of age on the biomechanics and physiology of gait. Clin Geriatr Med. 1996;12(4):659-78. doi: 10.1016/S0749-0690(18)30194-0. [PubMed: 8890109].
4. Parahyba MI, Veras R, Melzer D. [Disability among elderly women in Brazil]. Rev Saude Publica. 2005;39(3):383-90. Portuguese. doi: 10.1093/ageing/30.suppl_4.3. [PubMed:15997313].

5. Masud T, Morris RO. Epidemiology of falls. Age Ageing. 2001;30 Suppl 4:3-7. doi: 10.1093/ageing/30.suppl_4.3. [PubMed:11769786].

6. Fabricio SC, Rodrigues RA, da Costa MJ. [Falls among older adults seen at a Sao Paulo State public hospital: Causes and consequences]. Rev Saude Publica. 2004;38(1):93-9. Portuguese. doi: 10.1590/S003489102004000100013. [PubMed: 14963547].

7. Papaléo Netto M. O estudo da velhice no século XX: Histórico, definição do campo e termos básicos. In: Freitas EV, Py L, Néri AL, Cançado FAX, Gorzoni ML, Rocha SM, editors. Tratado de geriatria e gerontologia. 2nd ed. Rio de Janeiro: Guanabara Koogan; 2006. p. 2-12.

8. Siqueira FV, Facchini LA, Piccini RX, Tomasi E, Thumé E, Silveira DS, et al. Prevalência de quedas em idosos e fatores associados. Revista de Saúde Pública. 2007;41(5):749-56. Portuguese. doi: 10.1590/s003489102007000500009.

9. Waldron N, Hill AM, Barker A. Falls prevention in older adults - assessment and management. Aust Fam Physician. 2012;41(12):930-5. [PubMed: 23210114].

10. Tinetti ME, Speechley M, Ginter SF. Risk factors for falls among elderly persons living in the community. NEnglJMed.1988;319(26):1701-7. doi: 10.1056/NEJM198812293192604. [PubMed: 3205267].

11. Goudarzian M, Rahimi M, Karimi N, Samadi A, Ajudani R, Sahaf R, et al. Mobility, balance, and muscle strength adaptations to shortterm whole body vibration training plus oral creatine supplementation in elderly women. Asian J Sports Med. 2017;8(1). e36793. doi: 10.5812/asjsm.36793.

12. Overstall PW. The use of balance training in elderly people with falls. Rev Clin Gerontol. 2003;13(2):153-61. doi:10.1017/s0959259803013273.

13. Horak FB. Clinical assessment of balance disorders. Gait Posture. 1997;6(1):76-84. doi:10.1016/s0966-6362(97)00018-0.

14. Horak FB. Postural orientation and equilibrium: What do we need to know about neural control of balance to prevent falls? Age Ageing. 2006;35 Suppl 2:ii7-ii11. doi: 10.1093/ageing/aflo77. [PubMed: 16926210].

15. McComas J, Sveistrup H. Virtual reality applications for prevention, disability awareness, and physical therapy rehabilitation in neurology: Our recent work. J Neurol Phys Ther. 2002;26(2):55-61.

16. Bao T, Carender WJ, Kinnaird C, Barone VJ, Peethambaran G, Whitney SL, et al. Effects of long-term balance training with vibrotactile sensory augmentation among community-dwelling healthy older adults: A randomized preliminary study. J Neuroeng Rehabil. 2018;15(1):5. doi: 10.1186/s12984-017-0339-6. [PubMed: 29347946]. [PubMed Central: PMC5774163].

17. Thompson WR, Gordon NF, Pescatello LS. ACSM's guidelines for exercise testing and prescription. Philadelphia: Lippincott Williams \& Wilkins; 2009.

18. Dockx K, Alcock L, Bekkers E, Ginis P, Reelick M, Pelosin E, et al. Fall-prone older people's attitudes towards the use of virtual reality technology for fall prevention. Gerontology. 2017;63(6):590-8. doi: 10.1159/000479085. [PubMed: 28817828].

19. Neri SG, Cardoso JR, Cruz L, Lima RM, de Oliveira RJ, Iversen MD, et al. Do virtual reality games improve mobility skills and balance measurements in community-dwelling older adults? Systematic review and meta-analysis. Clin Rehabil. 2017;31(10):1292-304. doi: 10.1177/0269215517694677. [PubMed: 28933612].

20. Lange B, Chang CY, Suma E, Newman B, Rizzo AS, Bolas M. Development and evaluation of low cost game-based balance rehabilitation tool using the Microsoft Kinect sensor. Conf Proc IEEE Eng Med Biol Soc. 2011;2011:1831-4. doi: 10.1109/IEMBS.2011.6090521. [PubMed: 22254685].

21. Barcala L, Colella F, Araujo MC, Salgado ASI, Oliveira CS. [Balance analysis in hemiparetics patients after training with Wii Fit program]. Fisioterapia em Movimento. 2011;24(2):337-43. Portuguese. 
22. Taylor MJ, McCormick D, Shawis T, Impson R, Griffin M. Activitypromoting gaming systems in exercise and rehabilitation. J Rehabil Res Dev. 2011;48(10):1171-86. doi: 10.1682/JRRD.2010.09.0171. [PubMed: 22234662].

23. Clark RA, Pua YH, Oliveira CC, Bower KJ, Thilarajah S, McGaw R, et al. Reliability and concurrent validity of the Microsoft Xbox One Kinect for assessment of standing balance and postural control. Gait Posture. 2015;42(2):210-3. doi: 10.1016/j.gaitpost.2015.03.005. [PubMed: 26009500].

24. Perracini MR, Ramos LR. [Fall-related factors in a cohort of elderly community residents]. Rev Saude Publica. 2002;36(6):70916. Portuguese. doi: 10.1590/S0034-89102002000700008. [PubMed: 12488937].

25. Lin SI, Liao CF. Age-related changes in the performance of forward reach. Gait Posture. 2011;33(1):18-22. doi:10.1016/j.gaitpost.2010.09.013. [PubMed: 20951591].

26. Miyamoto ST, Lombardi Junior I, Berg KO, Ramos LR, Natour J. Brazilian version of the Berg balance scale. Braz J Med Biol Res. 2004;37(9):1411-21. doi: 10.1590/S0100-879X2004000900016. [PubMed: 15334208].

27. Berg KO, Wood-Dauphinee SL, Williams JI, Maki B. Measuring balance in the elderly: Validation of an instrument. Can J Public Health. 1992;83 Suppl 2:S7-11. [PubMed: 1468055].

28. Berg KO, Maki BE, Williams JI, Holliday PJ, Wood-Dauphinee SL. Clinical and laboratory measures of postural balance in an elderly population. Arch Phys Med Rehabil.1992;73(11):1073-80. [PubMed:1444775].

29. Mathias S, Nayak US, Isaacs B. Balance in elderly patients: The "getup and go" test. Arch Phys Med Rehabil. 1986;67(6):387-9. [PubMed: 3487300].

30. Podsiadlo D, Richardson S. The timed "Up \& Go": A test of basic functional mobility for frail elderly persons. J Am Geriatr Soc. 1991;39(2):142-8. doi: 10.1111/j.1532-5415.1991.tb01616.x. [PubMed: 1991946].

31. Diamantopoulos II, Clifford E, Birchall JP. Short-term learning effects of practice during the performance of the tandem Romberg test. Clin Otolaryngol Allied Sci. 2003;28(4):308-13. doi: 10.1046/j.13652273.2003.00710.x. [PubMed: 12871243].

32. Agrawal Y, Carey JP, Hoffman HJ, Sklare DA, Schubert MC. The modified Romberg Balance Test: Normative data in U.S. adults. Otol Neurotol. 2011;32(8):1309-11. doi: 10.1097/MAO.0b013e31822e5bee. [PubMed: 21892121]. [PubMed Central: PMC3190311].

33. Aveiro MC, Granito RN, Navega MT, Driusso P, Oishi J. Influence of a physical training program on muscle strength, balance and gait velocity among women with osteoporosis. Rev Bras Fisioter. 2006;10(4):441-8. doi:10.1590/s1413-35552006000400013.

34. Burdea G, Coiffet P. Virtual reality technology. Presence Teleoperators Virtual Environ. 2003;12(6):663-4. doi: 10.1162/105474603322955950.

35. Bisson E, Contant B, Sveistrup H, Lajoie Y. Functional balance and dual-task reaction times in older adults are improved by virtual reality and biofeedback training. Cyberpsychol Behav. 2007;10(1):16-23. doi: 10.1089/cpb.2006.9997. [PubMed: 17305444].

36. Donath L, Rossler R, Faude O. Effects of virtual reality training (exergaming) compared to alternative exercise training and passive control on standing balance and functional mobility in healthy community-dwelling seniors: A meta-analytical review. Sports Med. 2016;46(9):1293-309. doi: 10.1007/s40279-016-0485-1. [PubMed: 26886474].

37. Hara T, Shimada T. Effects of exercise on the improvement of the physical functions of the elderly. J Phys Ther Sci. 2007;19(1):15-26. doi: 10.1589/jpts.19.15.
38. Kimura T, Kobayashi H, Nakayama E, Hanaoka M. Effects of ag ing on gait patterns in the healthy elderly. Anthropological Science. 2007;115(1):67-72. doi: 10.1537/ase.060309.

39. Shumway-Cook A, Brauer S, Woollacott M. Predicting the probability for falls in community-dwelling older adults using the Timed Up \& Go Test. Phys Ther. 2000;80(9):896-903. [PubMed:10960937].

40. Okumiya K, Matsubayashi K, Nakamura T, Fujisawa M, Osaki Y, Doi Y, et al. The timed "up \& go" test is a useful predictor of falls in community-dwelling older people. J Am Geriatr Soc. 1998;46(7):92830. doi: 10.1111/j.1532-5415.1998.tb02737.x. [PubMed: 9670889].

41. Longridge NS, Mallinson AI. Clinical romberg testing does not detect vestibular disease. Otol Neurotol. 2010;31(5):803-6. doi 10.1097/MAO.0b013e3181e3deb2. [PubMed: 20502374].

42. Osti FR, de Souza CR, Teixeira LA. Improvement of balance stability in older individuals by on-water training. J Aging Phys Act. 2018;26(2):222-6. doi:10.1123/japa.2017-0041. [PubMed: 28657810].

43. Whitney SL, Poole JL, Cass SP. A review of balance instruments for older adults. Am J Occup Ther. 1998;52(8):666-71. doi 10.5014/ajot.52.8.666. [PubMed: 9739401].

44. Park SH, Lee YS. The diagnostic accuracy of the berg balance scale in predicting falls. West J Nurs Res. 2017;39(11):1502-25. doi: 10.1177/0193945916670894. [PubMed: 27784833].

45. Yesilyaprak SS, Yildirim MS, Tomruk M, Ertekin O, Algun ZC. Comparison of the effects of virtual reality-based balance exercises and conventional exercises on balance and fall risk in older adults living in nursing homes in Turkey. Physiother Theory Pract. 2016;32(3):191-201. doi: 10.3109/09593985.2015.1138009. [PubMed: 27049879].

46. Lesinski M, Hortobagyi T, Muehlbauer T, Gollhofer A, Granacher $U$. Effects of balance training on balance performance in healthy older adults: A systematic review and meta-analysis. Sports Med 2015;45(12):1721-38. doi: 10.1007/s40279-015-0375-y. [PubMed: 26325622]. [PubMed Central: PMC4656699].

47. Hernandez SS, Coelho FG, Gobbi S, Stella F. [Effects of physical activity on cognitive functions, balance and risk of falls in elderly patients with Alzheimer's dementia]. Rev Bras Fisioter. 2010;14(1):68-74. Portuguese. doi: 10.1590/S1413-35552010000100011. [PubMed: 20414564].

48. Pereira VV, Maia RA, Silva SM. The functional assessment Berg Balance Scale is better capable of estimating fall risk in the elderly than the posturographic Balance Stability System. Arq Neuropsiquiatr. 2013;71(1):5-10. doi: 10.1590/S0004-282X2013000100003. [PubMed: 23338160].

49. Jung Y, Yeh SC, McLaughlin. M , Rizzo AA, Winstein C. Threedimensional game environments for recovery from stroke. New York: Taylor and Francis; 2009.

50. Miller KJ, Adair BS, Pearce AJ, Said CM, Ozanne E, Morris MM. Effectiveness and feasibility of virtual reality and gaming system use at home by older adults for enabling physical activity to improve healthrelated domains: A systematic review. Age Ageing. 2014;43(2):188-95. doi: 10.1093/ageing/aft194. [PubMed: 24351549].

51. Clark RA, Pua YH, Fortin K, Ritchie C, Webster KE, Denehy L, et al. Validity of the Microsoft Kinect for assessment of postural control. Gait Posture. 2012;36(3):372-7. doi: 10.1016/j.gaitpost.2012.03.033. [PubMed: 22633015].

52. Llorens R, Alcaniz M, Colomer C, Navarro MD. Balance recovery through virtual stepping exercises using Kinect skeleton tracking: A follow-up study with chronic stroke patients. Stud Health Technol Inform. 2012;181:108-12. [PubMed: 22954838].

53. Bateni H. Changes in balance in older adults based on use of physical therapy vs the Wii Fit gaming system: A preliminary study. Physiotherapy. 2012;98(3):211-6. doi: 10.1016/j.physio.2011.02.004. [PubMed: 22898577]. 